\title{
Discrete-Time Sliding Mode Control Coupled with Asynchronous Sensor Fusion for Rigid-Link Flexible-Joint Manipulators
}

\author{
Guangyue Xue $\mathbb{D}^{1},{ }^{1}$ Xuemei Ren, ${ }^{2}$ Kexin Xing, ${ }^{3}$ and Qiang Chen ${ }^{3}$ \\ ${ }^{1}$ China Transport Telecommunications \& Information Center, Chaoyang District, Beijing 100011, China \\ ${ }^{2}$ School of Automation, Beijing Institute of Technology, Beijing 100081, China \\ ${ }^{3}$ College of Information Engineering, Zhejiang University of Technology, Hangzhou 310023, China
}

Correspondence should be addressed to Guangyue Xue; iviiivi@163.com

Received 4 March 2021; Revised 11 May 2021; Accepted 15 July 2021; Published 27 July 2021

Academic Editor: Xue-bo Jin

Copyright ( 2021 Guangyue Xue et al. This is an open access article distributed under the Creative Commons Attribution License, which permits unrestricted use, distribution, and reproduction in any medium, provided the original work is properly cited.

This paper proposes a novel discrete-time terminal sliding mode controller (DTSMC) coupled with an asynchronous multirate sensor fusion estimator for rigid-link flexible-joint (RLFJ) manipulator tracking control. A camera is employed as external sensors to observe the RLFJ manipulator's state which cannot be directly obtained from the encoders since gear mechanisms or flexible joints exist. The extended Kalman filter- (EKF-) based asynchronous multirate sensor fusion method deals with the slow sampling rate and the latency of camera by using motor encoders to cover the missing information between two visual samples. In the proposed control scheme, a novel sliding mode surface is presented by taking advantage of both the estimation error and tracking error. It is proved that the proposed controller achieves convergence results for tracking control in the theoretical derivation. Simulation and experimental studies are included to validate the effectiveness of the proposed approach.

\section{Introduction}

In many high-performance robotic manipulator applications, positioning of the end effector (or/and links) is critical as the ultimate goal is to track the desired trajectory. However, the challenge of achieving high accuracy and dynamic performance is increased due to the nonlinear flexibilities found in rigid-link flexible-joint (RLFJ) manipulators. Some researchers have designed observers to estimate states in the robot model, since link positions/ velocities are typically not measured in the industrial robot system. Nicosia and Tomei $[1,2]$ designed a controller combined with the observer which estimates motor positions or/and motor velocities for RLFJ manipulators. Dixon et al. [3] developed a globally adaptive output-feedback tracking controller for the RLFJ dynamics, which is based on the link velocity filter. Globally, output-feedback methods are not easily implemented in the real system because of the requirement of link position measurements which are not frequently measured in real systems. A controller was designed in $[4,5]$ by using a neural network $(\mathrm{NN})$ observer to estimate link and motor positions/velocities and dynamic parameters. But NN observer-based controllers do not take advantage of motor positions. Kalman filter (KF) and extended Kalman filter (EKF) are utilized to estimate positions of joints or/and end effectors, driving torque, and dynamic parameters for manipulators [6]. Lightcap and Banks [7] designed an EKF-RLFJ controller by using EKF to estimate link and motor positions/velocities. Garcła et al. [8] proposed compliant robot motion controllers by using EKF to fuse wrist force sensors, accelerometers, and joint position sensors. EKF-based sensor fusion was presented by JassemiZargani and Necsulescu [9] to estimate the acceleration for operational space control of a robotic manipulator. However, these reported EKF-based control methods do not discuss the case of asynchronous measurements from multirate sensors for RLFJ manipulators.

Observer-based sliding mode control (SMC) is one of the most important approaches to handle systems with uncertainties and nonlinearities [10]. For the RLFJ manipulator system, observer-based SMC of robot manipulators has been widely studied since the state of manipulators (e.g., 
acceleration velocity of joints) need not always be measured directly [11]. The terminal SMC (TSMC) is used in rigid manipulator control (e.g., robust TSMC and finite-time control) since it has some superior properties compared with SMC such as better tracking precision and fast error convergence [12-14]. In particular, the singularity problem of the TSMC was addressed in $[15,16]$. However, most of these papers use the continuous-time dynamic model of the manipulator. Indeed, discrete-time models exist widely in the real digital control system. It has been proved that the discrete-time SMC has technological advances in digital electronics, computer control, and robotic system. Corradinia and Orlando [17] presented a robust DSMC coupled with an uncertainty estimator designed for planar robotic manipulators. However, the flexibilities of joints are not involved in those controller designs.

To remedy such limitations, the paper proposes a novel controller, AMSFE-DTSMC, which is implemented based on DTSMC coupled with an asynchronous multirate sensor fusion estimator. The robotic multirate sensor unit contains vision and non-vision-based sensors whose sampling rate and processing time are different. In the proposed scheme, the delayed slow sampling vision measurement is treated as a kind of periodic "out-of-sequence" measurement (OOSM) [18] which is used to update the non-vision-based state estimation in an EKF-based asynchronous multirate sensor fusion algorithm. Using the position and velocity estimation from sensor fusion estimator, the DTSMC is designed by using a novel sliding surface which is implemented by considering estimation error and tracking error. The main contributions of this work are summarized as follows.

(i) We propose a novel tracking control scheme, AMSFE-DTSMC, which is based on the DTSMC coupled with the sensor fusion estimator for a RLFJ manipulator. The sliding surface of AMSFE-DTSMC is designed by utilzing estimation error and tracking control error.

(ii) We construct an asynchronous multirate measurement model for robotic sensors and design a sensor fusion to fuse such asynchronous multirate data for robotic state estimation.

This paper is organized as follows. Section 2 gives the problem formulation. In Section 3, the multirate sensor data fusion algorithm is presented. Section 4 designs a novel DTSMC for tracking control. The simulation and experimental studies are implemented in Section 5. The paper ends with conclusion about the proposed approach.

\section{Problem Formulation}

In this paper, a robotic manipulator system is given with the sensor unit including joint motor encoders and cameras fixed in the workspace. The tracking control scheme for RLFJ manipulators can be developed by using the robotic state estimate via multisensor fusion. The state of the robot is estimated by these sensors directly and indirectly; however, there are some limitations for a single sensor to obtain precise information. To fuse asynchronous multirate data from different sensors, the dynamic and sensor models are formulated in this section.

2.1. Discrete Rigid-Link Flexible-Joint Robot Model. The discrete dynamic model of an $n$-link RLFJ manipulator can be obtained by the minimization of the action functional suggested by Nicosia [2] as follows:

$$
\begin{aligned}
q(k+1) & =q(k)+T \dot{q}(k), \\
\dot{q}(k+1)-\dot{q}(k) & =M^{-1}(\bar{q}, \theta) T K_{s} \delta(k)-F_{L}(\bar{q}, q, \dot{q}, \theta) T, \\
M_{m}\left[\dot{q}_{m}(k+1)-\dot{q}_{m}(k)\right]+F_{m} \dot{q}_{m}(k) T & =T u(k)-K_{s} \delta(k) T, \\
\delta(k) & =q_{m}(k)-q(k),
\end{aligned}
$$

where $q(k), \dot{q}(k)$ and $q_{m}(k), \dot{q}_{m}(k)$ denote the position, velocity of the link and motor angles at $k$ time, respectively, $\bar{q}(k) \cong q(k)+T \dot{q}(k), T$ is the sampling interval, $M(\cdot)$ is the invertible inertia matrix which satisfies $\xi_{\min } \leq\|M\| \leq \xi_{\max }$, $F_{L}(\bar{q}, q, \dot{q}, \theta)=-M^{-1}(\bar{q}, \theta)[M(\bar{q}, \theta) \dot{q}-\quad M(q, \theta) \dot{q}-T f(q$, $\dot{q}), \theta], f(\cdot)$ represents centrifugal, Coriolis and gravitational forces; $K_{s}, M_{m}$ and $F_{m}$ are constant, diagonal, positivedefinite matrices representing joint stiffness, motor inertia, motor viscous friction, respectively; the joint deflection $\delta(k)$ is defined as the difference between the motor and link position and $u(k)$ denotes the motor torque; the unknown or varying dynamic parameter in the robotic model is defined as $\theta(k)$ which satisfy $\theta(k+1)-\theta(k)=T w_{\theta}(k)$, the dynamics uncertainties of the links and motors are modeled with random variables $w_{l}(k)$ and $w_{m}(k)$.

Define a state vector

$$
x(k)=\left[\begin{array}{lllll}
q(k)^{T} & \dot{q}(k)^{T} & q_{m}(k)^{T} & \dot{q}_{m}(k)^{T} & \theta(k)^{T}
\end{array}\right]^{T} .
$$

The dynamics in equations (1a)-(1d) can be transformed to a state space representation:

$$
x(k+1)=F_{k+1, k}(x(k), u(k))+\Gamma_{k} \omega(k+1, k),
$$

where 


$$
\begin{aligned}
F_{k+1, k}(x(k), u(k)) & =\left[\begin{array}{c}
q(k)+T \dot{q}(k) \\
\dot{q}(k)-F_{L}(k) T+M^{-1}(\bar{q})\left(K_{s} \delta(k) T\right) \\
q_{m}(k)+T \dot{q}_{m}(k) \\
M_{m}^{-1}\left[\dot{q}_{m}(k)-F_{m} \dot{q}_{m}(k) T-K_{s} \delta(k) T+T u(k)\right]
\end{array}\right], \\
\Gamma_{k} & =\left[\begin{array}{ccc}
0 & 0 & 0 \\
M(\bar{q}, \theta)^{-1} & 0 & 0 \\
0 & 0 & 0 \\
0 & M_{m}^{-1} & 0 \\
0 & 0 & I T
\end{array}\right], \\
\omega(k+1, k) & =\left[w_{l}(k)^{T}, w_{m}(k)^{T}, w_{\theta}(k)^{T}\right]^{T} .
\end{aligned}
$$

2.2. Measurement Model. The observation vector is given by

$$
h(x(k))=\left(q_{m}(k)^{T} \dot{q}_{m}(k)^{T} y(k)^{T}\right)^{T},
$$

where $q_{m}(k), \dot{q}_{m}(k)^{T}$ are the position and velocity of motor angles and $y(k)$ represents the position of the end effector in image space observed by a fixed camera.

By using the standard pinhole camera model, the mapping from the Cartesian space to image space is given by

$$
y(k)=I_{m}(r(k)),
$$

where $r(k)$ denotes the position of the end effector in the Cartesian space and $I_{m}(\cdot)$ is a transformation function from task space to image space. From the forward kinematics, the position relationship between robotic joints and end effector is described by a transform relationship as follows:

$$
r(k)=F_{k}(q(k)),
$$

where $r(k)$ is the position of the end effector at $k$ th time and $F_{k}(\cdot)$ is a transformation function from the joint space to task space.

From the equations (6) and (7), The joint position can be observed by a fixed camera:

$$
y(k)=I_{k}(q(k)),
$$

where $I_{k}(\cdot)=I_{m}\left(F_{k}(\cdot)\right)$ is the mapping from the joint space to image space. With the random noise $v(k)=\left[v_{1}^{T}\right.$ $\left.v_{2}^{T}(k) v_{3}^{T}(k)(k)\right]$, the measurement equation is given by

$$
z^{i}(k)=h_{k}^{i}(x(k))+\nu^{i}(k), \quad i=c, m,
$$

where $z^{i}(k)$ is the measurement of the state from different sensor, $i=c$ denotes the camera measurement, and $i=m$ represents the measurement from motors. We assume that the process noise $\omega(k)$ and measurement noise $\nu(k)$ are sampled from the independent and identically distributed white Gaussian noise which satisfies following equations at time $t_{k}$ :

$$
\left\{\begin{array}{l}
E\{\omega(k+1, k)\}=0, \\
E\{v(k)\}=0, \\
E\left\{\nu(j) \omega^{T}(k+1, k)\right\}=0, \\
E\left\{\omega(j+1, j) \omega_{N}^{T}(k+1, k)\right\}=Q(k) \delta_{j k}, \\
E\left\{\nu(j) \nu^{T}(k)\right\}=R(k) \delta_{j k} .
\end{array}\right.
$$

Since the measurement of the vision sensor at time $t_{k}$ is obtained from the visual image taken at time step $t_{\kappa}=t_{k}-t_{d}, t_{d}$ denotes the delay time. The relation of different sensors' sampling rates is given by

$$
s_{m}=l s_{c},
$$

where $s_{m}$ and $s_{c}$ denote the sampling rate of motor encoders and visual sensors, respectively, and $l$ is a positive integer.

We show the sampling rate difference between vision and non-vision measurements in Figure 1, where $l$ step lags of vision measurements are described. According to the characteristics of visual sensor, we treat the vision measurements as the periodic l-step lag out-of-sequence measurements.

Remark 1. The velocity of joints (or/and end effector) also can be observed by vision sensors, $\dot{y}(k)=J_{i k}(q(k)) \dot{q}(k)$, with the Jacobian matrix $J_{i k}(q)=J_{i}(r) J_{k}(q)$ mapping from the joint space to the image space. $J_{i}(r)$ is the image Jacobian matrix, and $J_{k}(q)$ denotes the Jacobian matrix from joint space to task space. However, the measurement of velocity is always impaired by the noisy image data with slow sampling rate and the dynamic uncertainties in the RLFJ manipulator system. Therefore, the velocity measurement in the image space is not utilized in the measurement model.

Remark 2. Assume that the camera can cover the entire workspace of the robot. With the prior knowledge of motion planning of the robot, it is basically assumed that there is an one-to-one mapping from the image space to joint space in the real robotic system. 


\section{Asynchronous Multirate Sensor Fusion Estimation}

According to the system model described in Section 2, the robotic link position can be estimated by using the multisensor system. For asynchronous multirate sensors, the sensor fusion method is designed to use the late measurements for updating the current estimated state to get a more accurate estimation in two steps:

Step 1: when the vision measurement is unavailable, the robotic state is estimated using the non-vision-based sensors which keep the real-time estimate by recovering the missing information between two vision samples.

Step 2: as the delayed vision measurement arrives at every vision sample at time $t=t_{l k}, l=1,2, \ldots$, the state should be re-estimated to cope with the limitations of other sensors in the absolute position measurement.

3.1. Estimation by Using Non-Vision Sensor Measurements. From Figure 1, before the $(k-1)$ th vision frame is available, we have the estimation of the state at time $t_{l k}$ using the nonvision sensor measurements:

$$
\begin{aligned}
& \widehat{x}(l k \mid l k)=E\left\{x(l k) \mid Z_{m}^{l k}\right\}, \\
& P(l k \mid l k)=\operatorname{cov}\left\{x(l k) \mid Z_{m}^{l k}\right\},
\end{aligned}
$$

where $Z_{l k}^{m}=\left\{z^{m}(1), z^{m}(2), \ldots, z^{m}(l k)\right\}$ represents all encoder motor measurements up to time $t_{l k}$. Using the extended Kalman filter (EKF), the estimation at time $t_{l k}$ via the motor encoder measurement is given as follows:

$$
\begin{aligned}
\hat{x}^{-}(l k \mid l k) & =F_{l k, l k-1}(\hat{x}(l k-1 \mid l k-1), u(l k)), \\
P(l k \mid l k-1) & =A_{l k} P(l k-1 \mid l k-1) A_{l k}^{T}+W_{l k} Q(l k-1) W_{l k}^{T},
\end{aligned}
$$

$$
K_{l k}=P(l k \mid l k-1) H_{l k}^{T}\left[H_{l k} \times P(l k \mid l k-1) H_{l k}^{T}+R(l k)\right]^{-1}
$$

$$
\hat{x}(l k \mid l k)=\hat{x}^{-}(l k \mid l k)+K_{l k}\left(z^{m}(l k)-h_{l k}^{m}\left(\hat{x}^{-}(l k \mid l k), 0\right)\right),
$$

$$
P(l k \mid l k)=\left(I-K_{l k} H_{l k}\right) P(l k \mid l k-1),
$$

where $A_{l k}, W_{l k}, H_{l k}$, and $V_{l k}$ are Jacobian matrices and $K_{l k}$ is the correction gain vector. According to above equations, the state and covariance estimates are updated with the measurement with a fast sampling rate.

3.2. Re-Estimation via Vision Sensor Measurements. Suppose that at the time $t_{l k}$ the vision measurement at time $t_{\kappa}=t_{k l-l}$ is obtained. A new estimation $\hat{x}\left(l k \mid \kappa^{+}\right)$is calculated using the information about the delayed $(k-1)$ th vision measurement $z^{c}(\kappa) . \quad \hat{x}\left(l k \mid \kappa^{+}\right)$is defined with $\mathbb{Z}_{l k}=\left\{Z_{l k}^{m}, z^{\mathcal{c}}(\kappa)\right\}:$

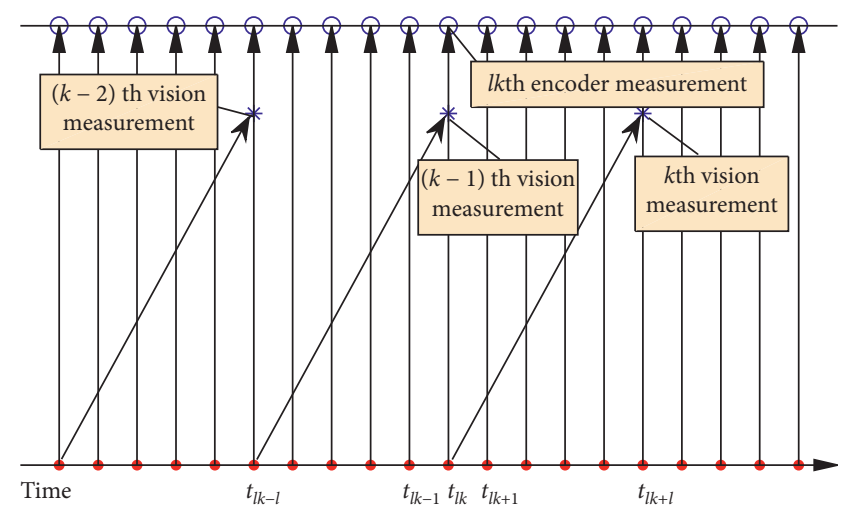

- Non-vision measurements

* Vision measurements

- System states

FIgURE 1: The schematic diagram of the relation between vision and non-vision measurements.

$$
\begin{aligned}
& \widehat{x}\left(l k \mid \kappa^{+}\right)=E\left\{x(l k) \mid \mathbb{Z}_{l k}\right\}, \\
& P\left(l k \mid \kappa^{+}\right)=\operatorname{cov}\left\{x(l k) \mid \mathbb{Z}_{l k}\right\} .
\end{aligned}
$$

The delayed measurement $z^{c}(\kappa)$ observed by vision measurement will be used to correct the accumulated estimation errors which are caused by sensors with fast sampling rate. The equations for updating the estimation $\hat{x}(l k \mid l k)$ with the delayed vision measurement $z^{c}(\kappa)$ are given by

$\hat{x}\left(l k \mid \kappa^{+}\right)=\widehat{x}(l k \mid l k)+P_{x z}(l k, \kappa \mid l k) \times P_{z z}(\kappa \mid l k)^{-1}[z(\kappa)-\widehat{z}(\kappa \mid l k)]$,

$P\left(l k \mid \kappa^{+}\right)=P(l k \mid l k)-P_{x z}(l k, \kappa \mid l k) \times P_{z z}(\kappa \mid l k)^{-1} P_{x z}(l k, \kappa \mid l k)^{T}$,

where $P_{x z}(l k, \kappa \mid l k)$ represents cross covariance between $\widehat{x}(l k \mid l k)$ and $\widehat{z}(\kappa \mid l k), P_{z z}(\kappa \mid l k)$ is the covariance of $\widehat{z}(\kappa \mid l k)$ which denotes the estimation of measurement at time $t_{\kappa}$, and

$$
\begin{aligned}
P_{x z}(l k, \kappa \mid l k) & =E\left\{\tilde{x}(l k) \tilde{z}^{c}(\kappa)^{T}\right\}, \\
P_{x z}(l k, \kappa \mid l k) & =E\left\{\tilde{z}^{c}(\kappa) \tilde{z}^{c}(\kappa)^{T}\right\}, \\
\widehat{z}(\kappa \mid l k) & =E\left\{z(\kappa) \mid Z_{l k}\right\},
\end{aligned}
$$

with $\left.\tilde{x}(l k)=x(l k)-\widehat{x}(l k \mid l k), \widetilde{z}^{c}(\kappa)=z^{c}(\kappa)-\widehat{z}(\kappa \mid l k)\right]^{T}$.

Using the EKF, $P_{x z}(l k, \kappa \mid l k), P_{x z}(l k, \kappa \mid l k)$, and $\widehat{z}(\kappa \mid l k)$ are obtained by assuming that the function $F_{k, k}(\cdot)$ is invertible. Define $F_{l k, \kappa}^{-1}(\cdot)=F_{\kappa, l k}(\cdot)$ which denotes the backward transition function to estimate the state back from $t_{l k}$ to $t_{\kappa}$. Since the previous state $x(\kappa)$ is not affected by present input signal $u(l k)$, we give the state relationship between $t_{\kappa}$ and $t_{l k}$ by

$$
x(\kappa)=F_{\kappa, l k}(x(l k)-\omega(l k, \kappa)),
$$

where the process noise $\omega(l k, \kappa)$ and covariance $Q(k l, \kappa)$ are calculated by 


$$
\begin{aligned}
\omega(l k, \kappa) \approx & \Gamma_{l k} \omega(l k, l k-1)+A_{l k, l k-1} \Gamma_{l k-1} \omega(l k-1, l k-2) \\
& +\cdots+A_{l k, l k-1} A_{l k-1, l k-2} \times \cdots \times A_{l k-l+2, l k-l+1} \Gamma_{l k-l+1} \omega(l k-l+1, l k-l), \\
Q(k l, \kappa)= & E\left\{\widetilde{\omega}(l k, \kappa) \widetilde{\omega}(l k, \kappa)^{T}\right\}, \\
\widetilde{\omega}(l k, \kappa)= & \omega(l k, \kappa)-\widehat{\omega}(l k, \kappa) .
\end{aligned}
$$

The estimation in equation (19) can be determined by

$$
\begin{aligned}
& \widehat{z}(\kappa \mid l k)=H_{l k}^{m}(\kappa) \widehat{x}(\kappa \mid l k), \\
& \widehat{x}(\kappa \mid l k)=F_{\kappa, l k}(\widehat{x}(l k \mid l k)-\widehat{\omega}(l k, \kappa \mid l k)) .
\end{aligned}
$$

To estimate the process noise as in equations (13)-(17), we have

$$
\widehat{\omega}(l k, \kappa \mid l k)=Q(l k, \kappa) H_{l k}^{T} P_{z z}(l k \mid l k-1)^{-1} \times(z(l k)-z(l k-1)) .
$$

Then, $P_{x z}(l k, \kappa \mid l k)$ and $P_{z z}(\kappa \mid l k)$ are obtained as follows:

$$
\begin{aligned}
P_{x z}(l k, \kappa \mid l k) & =\left(P(l k, \kappa \mid l k)-P_{x \omega}(l k, \kappa \mid l k)\right) A_{\kappa, l k}^{T} H(\kappa)^{T}, \\
P_{z z}(\kappa \mid l k) & =H(\kappa) P(\kappa \mid l k) H(\kappa)^{T}+R(\kappa),
\end{aligned}
$$

where the covariances $P(\kappa \mid l k)$ and $P_{x \omega}(l k, \kappa \mid l k)$ are derived as follows:

$$
\begin{aligned}
P(\kappa \mid l k) & =A_{\kappa, l k}\left(P(l k \mid l k)+P_{\omega \omega}(\kappa \mid l k)-P_{x \omega}(l k, \kappa \mid l k)^{T}\right) A_{\kappa, l k}^{T}-P_{x \omega}(l k, \kappa \mid l k), \\
P_{\omega \omega}(\kappa \mid l k) & =Q(l k, \kappa)-Q(l k, \kappa) H_{l k}^{T} \times P_{z z}(l k \mid l k-1)^{-1} H_{l k} Q(l k, \kappa)^{T}, \\
P_{x \omega}(l k, \kappa \mid l k) & =Q(l k, \kappa)-P(l k \mid l k-1) H_{l k}^{T} \times P_{z z}(l k \mid l k-1)^{-1} H_{l k} Q(l k, \kappa)^{T} .
\end{aligned}
$$

3.3. Summary of the Fusion Estimate Method. The state of a RLFJ manipulator is estimated using the indirect measurements from asynchronous multirate sensors. The sensor fusion estimate can be implemented in the practical application using a switching mechanism in accordance with sampling time. As shown in Figure 1, the update equations are chosen at the different sampling times. The state estimation can be given by

$$
\widehat{x}_{F}(k \mid k)= \begin{cases}F_{k, k-1}\left(\hat{x}^{-}(k \mid k)+K_{k}\left[z^{m}(k)-h_{k}^{m}\left(\hat{x}^{-}(k \mid k), 0\right)\right],\right. & t \neq t_{l k}, \\ \widehat{x}(k \mid k)+P_{x z}(k, k-l \mid k) P_{z z}^{-1}(k-l \mid k)\left(z^{c}(k-l)-\widehat{z}(k-l \mid k)\right), & t=t_{l k} .\end{cases}
$$

Remark 3. The exponential convergence of the sensor fusion estimate can be proved in the similar way which is presented in [19], which has more detailed conclusion on the stability.

\section{Rigid-Link Flexible-Joint Tracking Control}

In this section, the discrete-time terminal sliding mode tracking control-based fusion estimation (AMSFE-DTSMC) is presented for rigid-link flexible manipulators whose state is estimated by the sensor fusion method described in the previous section. The controller is designed by using both position and velocity of the link from the sensor fusion estimate. To design the novel controller, the model in (1a)-(1d) can written as

$$
\begin{aligned}
& q(k+1)=q(k)+T \dot{q}(k), \\
& \dot{q}(k+1)=\dot{q}(k)+M^{-1}(\bar{q}, \theta) T u(k)-\mathbb{F}\left(\bar{q}, q, \dot{\bar{q}}_{m}, \dot{q}_{m}, T, \theta\right),
\end{aligned}
$$

where $\mathbb{F}\left(\bar{q}, q, \dot{\bar{q}}_{m}, \dot{q}_{m}, T, \theta\right)=F_{L}(\bar{q}, q, \dot{q}, \theta) T+M^{-1}(\bar{q}, \theta)\left[M_{m}\right.$ $\left.\left(\dot{q}_{m}(k+1)-\dot{q}_{m}(k)\right)+F_{m} \dot{q}_{m}(k)\right]$ denotes the variable which includes dynamic parameters of links and motors.

In order to formulate the tracking control, define the tracking error $\tilde{q}_{t}(k)$ and estimation error $\tilde{q}_{e}(k)$ at time $t_{k}$ as

$$
\begin{aligned}
& \tilde{q}_{t}(k)=q(k)-q_{d}(k), \\
& \tilde{q}_{e}(k)=q(k)-\widehat{q}(k),
\end{aligned}
$$

where $q_{d}(k)$ is the desired position and $\hat{q}(k)$ denotes the estimation position.

Define the reference velocity for tracking and estimation:

$$
\begin{aligned}
\dot{q}_{t}(k) & =\dot{q}_{d}(k)+\Lambda_{t}\left(q_{d}(k)-\widehat{q}(k)\right) \\
& =\dot{q}_{d}(k)-\Lambda_{t}\left(\widetilde{q}_{t}(k)-\widetilde{q}_{e}(k)\right), \\
\dot{q}_{e}(k) & =\dot{\vec{q}}(k)-\Lambda_{e}(q(k)-\widehat{q}(k)) \\
& =\dot{\hat{q}}(k)-\Lambda_{e} \widetilde{q}_{e}(k),
\end{aligned}
$$

where $\dot{\hat{q}}(k)$ denotes the estimation of $\dot{q}(k)$ and $\Lambda_{t}, \Lambda_{e}$ are constants and diagonal matrices. 
Define the filtered variables including the estimation error:

$$
\widetilde{\dot{q}}_{t}(k)=\dot{q}(k)-\dot{q}_{t}(k)=\dot{\tilde{q}}_{t}(k)+\Lambda_{t}\left(\tilde{q}_{t}(k)-\tilde{q}_{e}(k)\right) .
$$

Consider the discrete terminal sliding surface as follows:

$$
s(k)=\tilde{\dot{q}}_{t}(k)+\lambda \widetilde{q}_{t}(k)^{p},
$$

where $\lambda$ is a positive constant diagonal parameter matrix and $p=p_{1} / p_{2}$, in which $p_{1}$ and $p_{2}$ are positive odd integers satisfying $p_{2}>p_{1}$. Motivated by the reaching law presented by Weibing Gao et al. in [20], we use the reaching law for exponential discrete sliding mode control as follows:

$$
s(k+1)=(I-T h) s(k)-T \varepsilon \operatorname{sgn}(s(k)),
$$

where $\operatorname{sgn}(\cdot)$ is the signum function, $\varepsilon>0$, and $h>0$.

Since system states cannot be measured directly, parameters which contain variables $q(k), \dot{q}(k)$, and $\theta$ need to be estimated in the controller design. Assume that the estimate error and uncertainties are bounded, and we have

$$
\begin{aligned}
M(\bar{q}, \theta) & =\widehat{M}(\bar{q}, \theta)+\Delta M(\bar{q}, \theta), \\
\mathbb{F}\left(\bar{q}, q, \dot{\bar{q}}_{m}, \dot{q}_{m}, T, \theta\right) & =\widehat{M} \mathbb{F}\left(\bar{q}, q, \dot{\bar{q}}_{m}, \dot{q}_{m}, T, \theta\right)+\Delta \mathbb{F}\left(\bar{q}, q, \dot{\bar{q}}_{m}, \dot{q}_{m}, T, \theta\right),
\end{aligned}
$$

where $\dot{\bar{q}}_{m}$ denotes $\dot{q}_{m}(k+1) ; \quad \widehat{M}(\bar{q}, \theta)=M(\hat{\bar{q}}, \hat{\theta}) \quad$ and $\widehat{\mathbb{F}}\left(\bar{q}, q, \dot{\bar{q}}_{m}, \dot{q}_{m}, T, \theta\right)=\mathbb{F}\left(\overline{\bar{q}}, \hat{q}, \dot{\bar{q}}_{m}, \hat{\dot{q}}_{m}, T, \theta\right)$ represent the estimation of dynamic parameters; and $\Delta M(\bar{q}, \theta)$ and $\Delta \mathbb{F}\left(\bar{q}, q, \dot{\bar{q}}_{m}, \dot{q}_{m}, T, \theta\right)$ are bounded variables including the estimation error and system uncertainties, which satisfy

$$
\begin{array}{r}
\|\Delta M(\bar{q}, \theta)\| \leq \delta_{M}, \\
\left\|\Delta \mathbb{F}\left(\bar{q}, q, \dot{\bar{q}}_{m}, \dot{q}_{m}, T, \theta\right)\right\| \leq \delta_{\mathbb{F}},
\end{array}
$$

where $\delta_{M}$ and $\delta_{F}$ are constants.

Theorem 1. Consider the rigid-link flexible-joint manipulator system described by equations (29a) and (29b) and the discrete sliding manifold described by equation (33); by using the reaching law in equation (34), stable control law is designed as

$$
\begin{aligned}
u(k)= & \frac{\hat{M}(\bar{q}, \theta)}{T}\left[\widehat{\mathbb{F}}\left(\bar{q}, q, \dot{\bar{q}}_{m}, \dot{q}_{m}, T, \theta\right)+\dot{q}_{t}(k)-\dot{q}_{t}(k-1)+\lambda \widetilde{q}_{t}^{p}(k)\right. \\
& -\lambda\left[T\left(\tilde{\dot{q}}_{t}(k)-\Lambda_{t} \widetilde{q}_{t}(k)+\Lambda_{t} \widetilde{q}_{e}(k)\right)+\widetilde{q}_{t}(k)\right]^{p} \\
& -T(h s(k)+\varepsilon(k) \operatorname{sgn}(s(k)))],
\end{aligned}
$$

where $\varepsilon$ and h are positive diagonal matrices which satisfy the following inequations:

$$
\begin{aligned}
\varepsilon(k)> & \frac{1}{T} \xi_{\mathbb{M}}\left\|\Delta \dot{q}_{t}(k)-\lambda\left[T\left(\tilde{q}_{t}(k)-\Lambda_{t} \widetilde{q}_{t}(k)+\Lambda_{t} \widetilde{q}_{e}(k)\right)+\widetilde{q}_{t}(k)\right]^{p}-\lambda \widetilde{q}_{t}^{p}(k)\right\| \\
& +\frac{1}{T} \xi_{\mathbb{F}}+h\|s(k)\|, \\
T< & \frac{2\|s(k)-\Delta \mathbb{F}\|}{h\|s(k)\|+\|\varepsilon(k)\|} .
\end{aligned}
$$

Proof. Substituting control law (37) in the rigid-link flexible-joint system equations (29a) and (29b), the error dynamics are obtained:

$$
\begin{aligned}
\tilde{q}_{t}(k+1)= & \tilde{q}_{t}(k)+T\left(\tilde{\dot{q}}_{t}(k)-\Lambda_{t} \widetilde{q}_{t}(k)+\Lambda_{t} \widetilde{q}_{e}(k)\right), \\
\tilde{\dot{q}}_{t}(k+1)= & \tilde{\dot{q}}_{t}(k)+\lambda \widetilde{q}_{t}(k)^{p} \\
& -\lambda\left[T\left(\tilde{\dot{q}}_{t}(k)-\Lambda_{t} \widetilde{q}_{t}(k)+\Lambda_{t} \widetilde{q}_{e}(k)\right)+\widetilde{q}_{t}(k)\right]^{p} \\
& +\left(M^{-1} \widehat{M}-I\right)\left[\widehat{\mathbb{F}}+\Delta \dot{q}_{t}(k)-\lambda\left[T \left(\widetilde{\dot{q}}_{t}(k)\right.\right.\right. \\
& \left.\left.\left.-\Lambda_{t} \widetilde{q}_{t}(k)+\Lambda_{t} \widetilde{q}_{e}(k)\right)+\widetilde{q}_{t}(k)\right]^{p}-\lambda \widetilde{q}_{t}^{p}(k)\right] \\
& +M^{-1} \widehat{M}[\Delta \mathbb{F}-T(h s(k)+\varepsilon(k) \operatorname{sgn}(s(k)))] .
\end{aligned}
$$


For simplicity, define $\mathbb{M}=M^{-1} \widehat{M}$ which is invertible and $\mathbb{M}^{-1}=\widehat{M}^{-1} M$. Substituting (40) and (41) into (34) yields

$$
\begin{aligned}
s(k+1)= & s(k)+\mathbb{M}[-T(h s(k)-\varepsilon(k) \operatorname{sgn}(s(k)))] \\
& +\mathbb{M} \widehat{\mathbb{F}}-\mathbb{F}+(\mathbb{M}-I)\left[\Delta \dot{q}_{t}(k)-\lambda \widetilde{q}_{t}^{p}(k)\right. \\
& \left.-\lambda\left[T\left(\tilde{\dot{q}}_{t}(k)-\Lambda_{t} \widetilde{q}_{t}(k)+\Lambda_{t} \widetilde{q}_{e}(k)\right)+\widetilde{q}_{t}(k)\right]^{p}\right] .
\end{aligned}
$$

Stability conditions for the discrete sliding mode control are given by Sarpturk [21]:

$$
\begin{aligned}
\varepsilon(k)> & \frac{1}{T}\left(I-\mathbb{M}^{-1}\right)\left[-\lambda\left[T \left(\tilde{\dot{q}}_{t}(k)-\Lambda_{t} \widetilde{q}_{t}(k)\right.\right.\right. \\
& \left.\left.\left.+\Lambda_{t} \widetilde{q}_{e}(k)\right)+\widetilde{q}_{t}(k)\right]^{p}-\lambda \widetilde{q}_{t}^{p}(k)\right]+\frac{1}{T}\left(I-\mathbb{M}^{-1}\right) \Delta \dot{q}_{t}(k)+\frac{1}{T}\left(\widehat{\mathbb{F}}-\mathbb{M}^{-1} \mathbb{F}\right)-h s(k),
\end{aligned}
$$

Combining (42) and (43a), we have

$$
\begin{aligned}
s^{T} & {[-\mathbb{M} T[h s(k)+\varepsilon(k) \operatorname{sgn}(s(k))]+\mathbb{M} \widehat{\mathbb{F}}-\mathbb{F}} \\
& +(\mathbb{M}-I)\left[\Delta \dot{q}_{t}(k)-\lambda\left(T \tilde{\dot{q}}_{t}(k)-T \Lambda_{t} \widetilde{q}_{t}(k)\right.\right. \\
& \left.\left.\left.+T \Lambda_{t} \widetilde{q}_{e}(k)+\widetilde{q}_{t}(k)\right)^{p}-\lambda \widetilde{q}_{t}^{p}(k)\right]\right]<0 .
\end{aligned}
$$

If $s(k)>0, \operatorname{sgn}(s(k))=1$, and $s(k+1)-s(k)<0$, the sliding gain lower bound can be given as
Employ $\xi_{\mathbb{M}}$ and $\quad \xi_{\mathbb{F}}$ which satisfy $\left\|I-\mathbb{M}^{-1}\right\|<\xi_{\mathbb{M}}$, $\left\|\hat{\mathbb{F}}-\mathbb{M}^{-1} \mathbb{F}\right\|<\xi_{\mathbb{F}}$, we can obtain $\varepsilon(k)$ as follows:

$$
\begin{aligned}
\varepsilon(k)> & \frac{1}{T} \xi_{\mathbb{M}} \| \Delta \dot{q}_{t}(k)-\lambda\left[T\left(\tilde{\dot{q}}_{t}(k)-\Lambda_{t} \tilde{q}_{t}(k)+\Lambda_{t} \tilde{q}_{e}(k)\right)\right. \\
& \left.+\tilde{q}_{t}(k)\right]^{p}-\lambda \widetilde{q}_{t}^{p}(k)\left\|+\frac{1}{T} \xi_{\mathbb{F}}+h\right\| s(k) \| .
\end{aligned}
$$

$$
\begin{aligned}
s(k+1)= & s(k)+\mathbb{M} T[\varepsilon(k)-h s(k)]+\mathbb{M} \widehat{\mathbb{F}}-\mathbb{F} \\
& +(\mathbb{M}-I)\left[\Delta \dot{q}_{t}(k)-\lambda \widetilde{q}_{t}^{p}(k)-\lambda\left[T\left(\widetilde{\dot{q}}_{t}(k)-\Lambda_{t} \widetilde{q}_{t}(k)+\Lambda_{t} \widetilde{q}_{e}(k)\right)+\widetilde{q}_{t}(k)\right]^{p}\right] .
\end{aligned}
$$

Employ $\varepsilon(k)$ given by equation (46), and we have

$$
\begin{aligned}
s(k+1)-s(k)> & \mathbb{M} T h[\|s(k)\|-s(k)] \\
& +\left[\mathbb{M} \xi_{\mathbb{F}}-\mathbb{M} \widehat{\mathbb{F}}+\mathbb{F}\right] \\
& +\mathbb{M} \xi_{\mathbb{M}} \| \Delta \dot{q}_{t}(k)-\lambda\left[T \widetilde{\dot{q}}_{t}(k)-T \Lambda_{t} \widetilde{q}_{t}(k)\right. \\
& \left.+T \Lambda_{t} \widetilde{q}_{e}(k)+\widetilde{q}_{t}(k)\right]^{p}-\lambda \widetilde{q}_{t}^{p}(k) \| \\
& -(\mathbb{M}-I)\left[\Delta \dot{q}_{t}(k)-\lambda\left(T \tilde{\dot{q}}_{t}(k)-T \Lambda_{t} \tilde{q}_{t}(k)\right.\right. \\
& \left.\left.+T \Lambda_{t} \tilde{q}_{e}(k)+\widetilde{q}_{t}(k)\right)^{p}-\lambda \widetilde{q}_{t}^{p}(k)\right],
\end{aligned}
$$


where $s(k+1)-s(k)>0$ is obtained by adopting $\varepsilon(k)$ according to equation (46). Therefore, equation (46) satisfied the Sarpturk stability (43a).

Assuming $\mathbb{M}=I$, Sarpturk condition (3) is formulated by combining equations (42) and (43b):

$$
\begin{aligned}
s(k+1)+s(k)= & 2 s(k)-\Delta \mathbb{F} \\
& -T[h s(k)+\varepsilon(k) \operatorname{sgn}(s(k))] .
\end{aligned}
$$

If $s(k)<0, s(k+1)+s(k)<0$, we have

$$
2 s(k)-T(h s(k)-\varepsilon(k))-\Delta \mathbb{F}<0 .
$$

Else if $s(k)>0, s(k+1)+s(k)>0$, we have

$$
2 s(k)-T(h s(k)+\varepsilon(k))-\Delta \mathbb{F}>0 .
$$

The sampling time $T$ which satisfies the following equation can guarantee the condition in (43b):

$$
0<T<\frac{2\|s(k)-\Delta \mathbb{F}\|}{h\|s(k)\|+\|\varepsilon(k)\|} .
$$

From (52), we know that the stability conditions of DTSMC given by (43a) and (43b) are guaranteed by chosen parameters $\varepsilon$ and $h$ defined in (38) and (39). Theorem 1 is proved.

\section{Simulation and Experimental Studies}

5.1. Simulation Study. The results obtained from the simulation of proposed control scheme on a two-link RLFJ manipulators shown in Figure 2 are presented in this section. In the simulation, the aim is make the RLFJ manipulator track desired trajectories $q_{1, d}=\sin (\pi t)$ and $q_{2, d}=\cos (\pi t)$ from the initial position $\left[q_{1 i} q_{2 i}\right]=[0.5,0.5]$. The robotic dynamic parameters are given by

$$
\begin{aligned}
M(q) & =\left[\begin{array}{ll}
M_{11} & M_{12} \\
M_{21} & m_{2} l_{2}^{2}
\end{array}\right], \\
g(q) & =\left[\begin{array}{c}
g_{1} \\
\left.-m_{2} g l_{2} \sin \left(q_{1}+q_{2}\right)\right)
\end{array}\right], \\
C & =\left[\begin{array}{c}
-m_{2} l_{1} l_{2} \sin \left(q_{2}\right)\left(2 \dot{q}_{1} \dot{q}_{2}+\dot{q}_{2}^{2}\right) \\
-m_{2} l_{1} l_{2} \sin \left(q_{2}\right) \dot{q}_{1} \dot{q}_{2},
\end{array}\right], \\
M_{11} & \left.=m_{1}+m_{2}\right) l_{1}^{2}+m_{2} l_{2}^{2}+2 m_{2} l_{1} l_{2} \cos \left(q_{2}\right), \\
M_{12} & =M_{21}=m_{2} l_{2}^{2}+m_{2} l_{1} l_{2} \cos \left(q_{2}\right), \\
g_{1} & =-\left(m_{1}+m_{2}\right) g l_{1} \sin \left(q_{1}\right)-m_{2} g l_{2} \sin \left(q_{1}+q_{2}\right),
\end{aligned}
$$

where $m_{i}$ and $l_{i}$ are given in Table 1. For $i=1,2, m_{i}$ and $l_{i}$ denote mass and length of the ith link. $k_{m}=\operatorname{diag}\{10,10\} \mathrm{Num} / \mathrm{rad}, M_{m}=$ $\operatorname{diag}\{0.5,0.5\} \mathrm{kg} \cdot \mathrm{m}^{2}, F_{m}=\operatorname{diag}\{4,0.5\} \mathrm{Nm} \cdot \mathrm{sec} / \mathrm{rad}$.

In order to demonstrate the influence of estimation for tracking performance, a DTSMC controller without estimation is also simulated. The DTSMC control law is given by

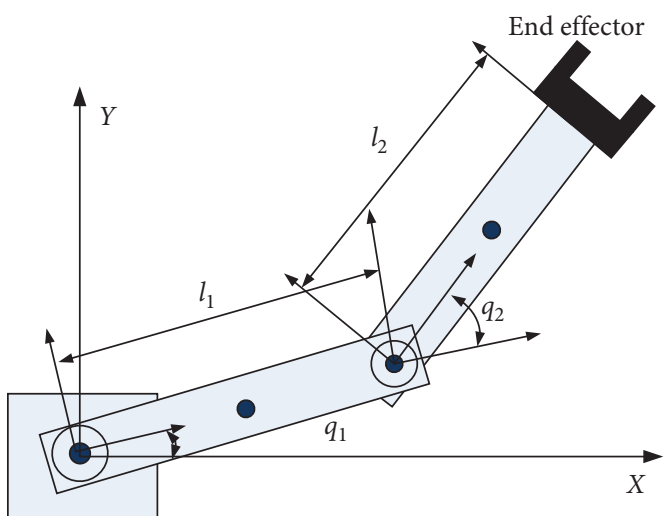

Figure 2: The schematic diagram of robotic manipulator with kinematics parameters.

TABLE 1: The manipulator parameters.

\begin{tabular}{lccc}
\hline Link $_{i}$ & $m_{i}(\mathrm{~kg})$ & $l_{i}(\mathrm{~cm})$ & $q_{i}$ \\
\hline 1 & 2.5 & 35 & $q_{1}\left(0^{\circ} \sim 180^{\circ}\right)$ \\
2 & 3 & 20 & $q_{2}\left(-160^{\circ} \sim 160^{\circ}\right)$ \\
\hline
\end{tabular}

$$
\begin{aligned}
u(k)= & \frac{\widehat{M}}{T}\left[\widehat{\mathbb{F}}+\left(\dot{q}_{d}(k)-\dot{q}_{d}(k-1)+\lambda \widetilde{q}_{t}^{p}(k)\right.\right. \\
& \left.-\lambda\left[T \dot{\tilde{q}}_{t}(k)+\widetilde{q}_{t}(k)\right]^{p}\right]-h s(k)-\varepsilon(k) \operatorname{sgn}(s(k)),
\end{aligned}
$$

with a sliding surface $s(k)=\dot{\tilde{q}}_{t}(k)+\lambda \widetilde{q}_{t}(k)^{p}$ which is different from proposal sliding mode surface.

Parameters of the proposed controller and DTSMC are selected as $T=0.001 \mathrm{~s}, h=\operatorname{diag}\{2,2\}, \lambda=\operatorname{diag}\{1.5,1.5\}$, $\Lambda_{t}=\operatorname{diag}\{5,5\}$, and $\varepsilon=\operatorname{diag}\{25,25\} \cdot \theta(k)=0.15$ is chosen as a constant model parameter whose initial value is $\theta(k)=0$, $p=3, q=5, \xi_{\mathbb{M}}=\operatorname{diag}\{3,3\}$, and $\xi_{\mathbb{F}}=\operatorname{diag}\{1,1\}$. In the simulation, as shown in Figure 3, we assume that the end effector is observed by a fixed camera whose delayed measurements are used directly to calculate the joint position. The delayed time of slow measurement is $0.125 \mathrm{~s}$. The process and measurement noise are chosen as $Q=0.01 I$ and $R=0.1 I$. The initial position value of joints (joint1, joint 2 ) for tracking control is given by ( $0.5 \mathrm{rad}, 0.5 \mathrm{rad})$, and for fusion estimator, it is given by ( $0.9 \mathrm{rad}, 0.9 \mathrm{rad})$. The initial velocity value for tracking control is given by $(0,0)$, and for estimation, it is given by $(0.25 \mathrm{rad} / \mathrm{s}, 0)$.

The estimate errors of position and velocity are plotted in Figure 4 . The estimate errors of parameter $\theta$ are shown in Figure 5. The position tracking of the proposed method for two links is shown in Figures 6 and 7 where the comparative result of SMC without fusion estimator is also plotted. In conclusion, the simulation results clearly indicate that the proposed approach guarantees the convergence of tracking errors and has better tracking accuracy.

5.2. Experimental Study. To validate the applicability of the proposed control schemes, a single-link flexible-joint 

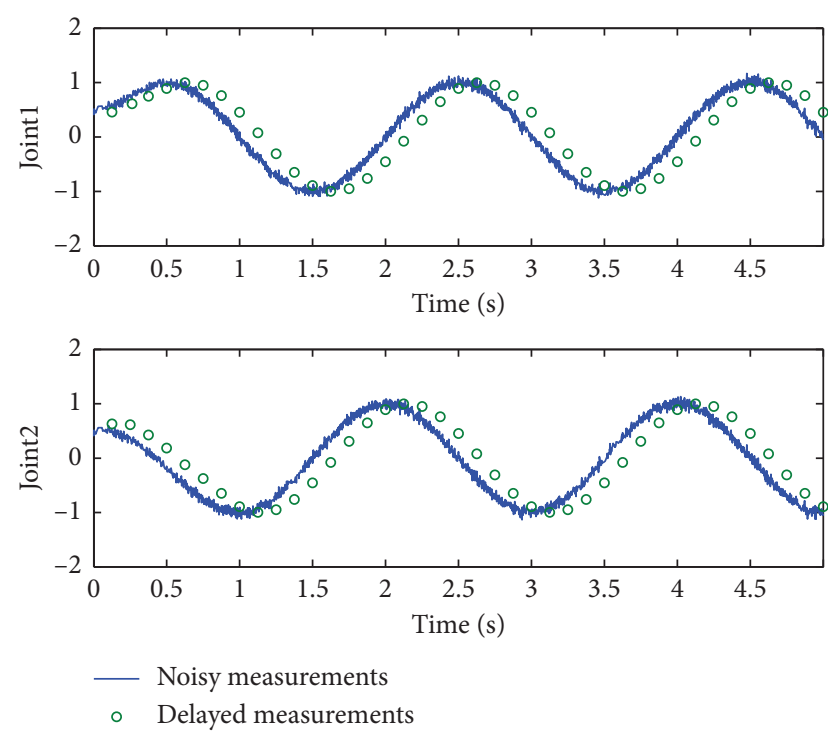

FIgURE 3: Asynchronous multirate sensor measurements.

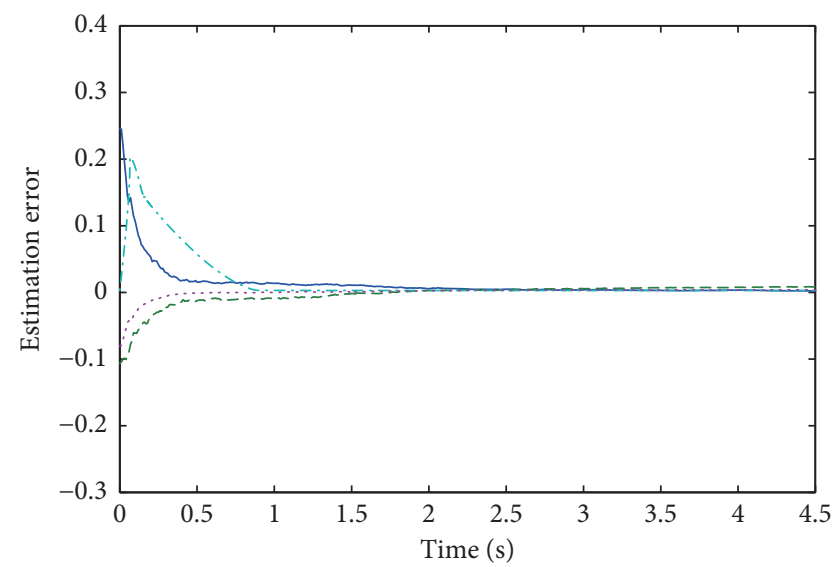

_ Velocity estimation error of link1

- - - Position estimation error of link1

Velocity estimation error of link2

.... Position estimation error of link2

FIgURE 4: The estimation errors of $q_{1}, \dot{q}_{1}, q_{2}, \dot{q}_{2}$.

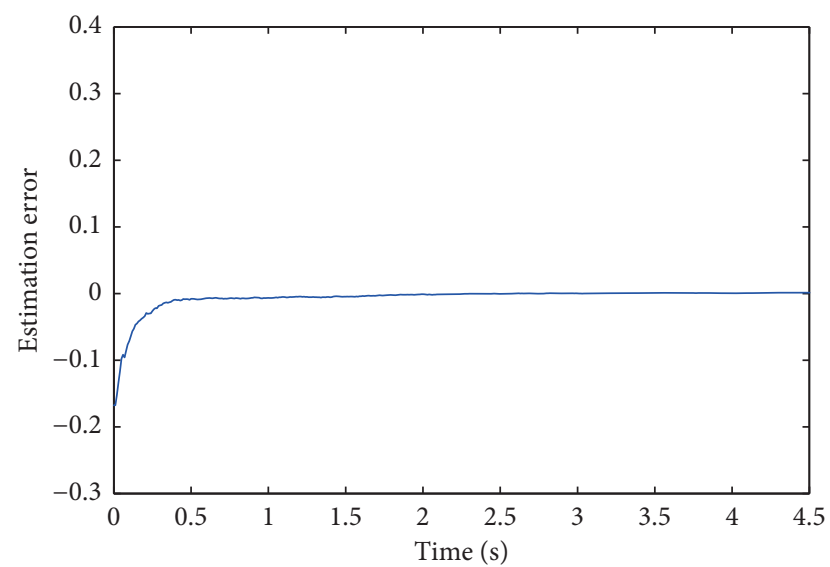

FIgURE 5: The estimation error of $\theta$.

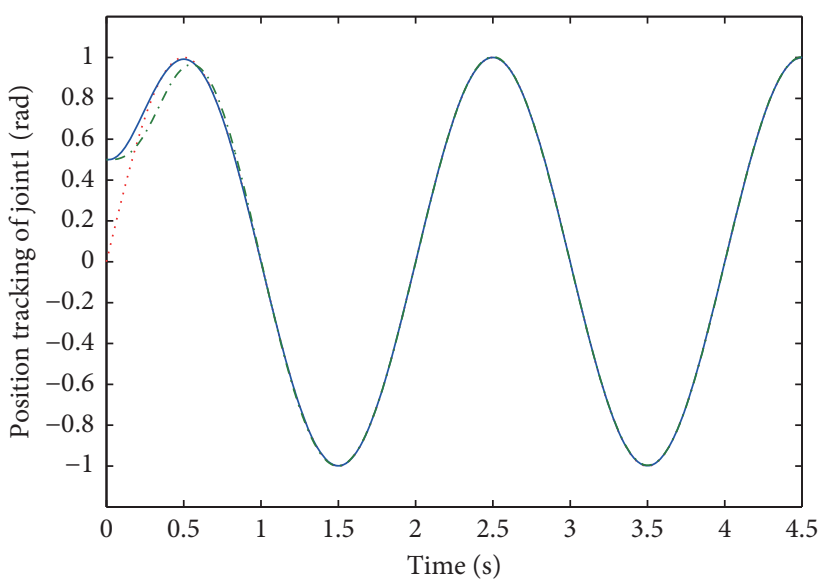

Reference trajectory

— Proposed method

-..- SMC

FIGURE 6: Comparative control performances of joint 1 between the proposed method and SMC.

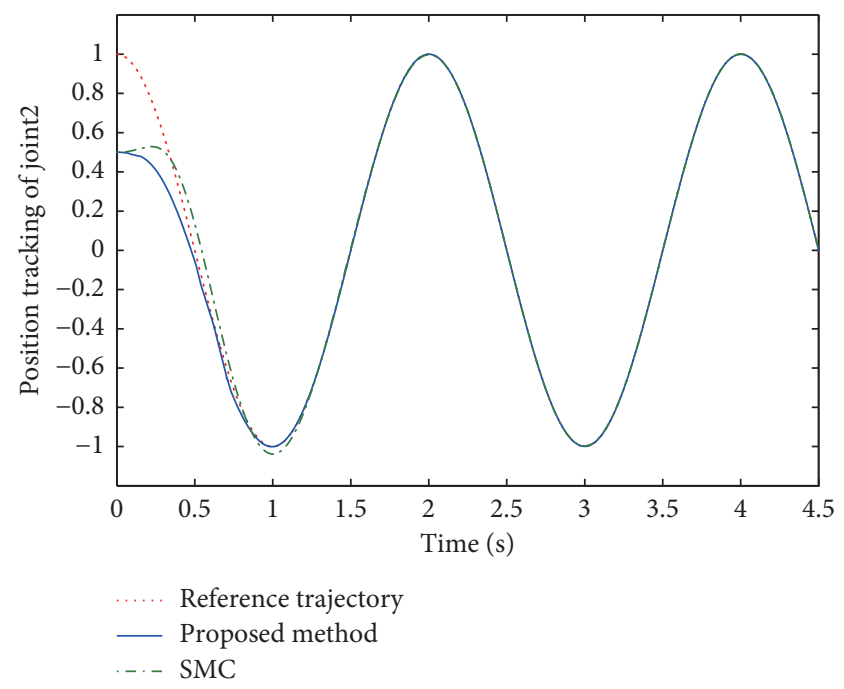

FIGURE 7: Comparative control performances of joint 2 between the proposed method and SMC.

manipulator with a fixed camera is employed as the experimental plant which is shown in Figure 8. The aim is to make the end effector move along the desired trajectory $x_{d}=l_{1} \cos \left(q_{d}\right), y_{d}=l_{1} \sin \left(q_{d}\right)$ with $q_{d}=(0.4-t) / 75$, and the initial position of joint is $q_{i}=0.35 \mathrm{rad}$. Parameters of the single-link robot are measured offline: $l_{1}=0.6 \mathrm{~m}$ and $m_{1}=10 \mathrm{~kg}$; the measurement errors are $\Delta l_{1}=0.05 \mathrm{~m}$ and $\Delta m_{1}=0.1 \mathrm{~kg}$.

To observe the state of end effector, the calibrated camera is fixed perpendicular to the robot notion plane. The coordinate relation of Cartesian space and image space is shown in Figure 9. Camera measurements are obtained from the image sequences shown in Figure 10. We define the position in the image coordinate $y(k)=\left[x_{m}(k), y_{m}(k)\right]$ given in Figure 11, and the position in Cartesian coordinate 


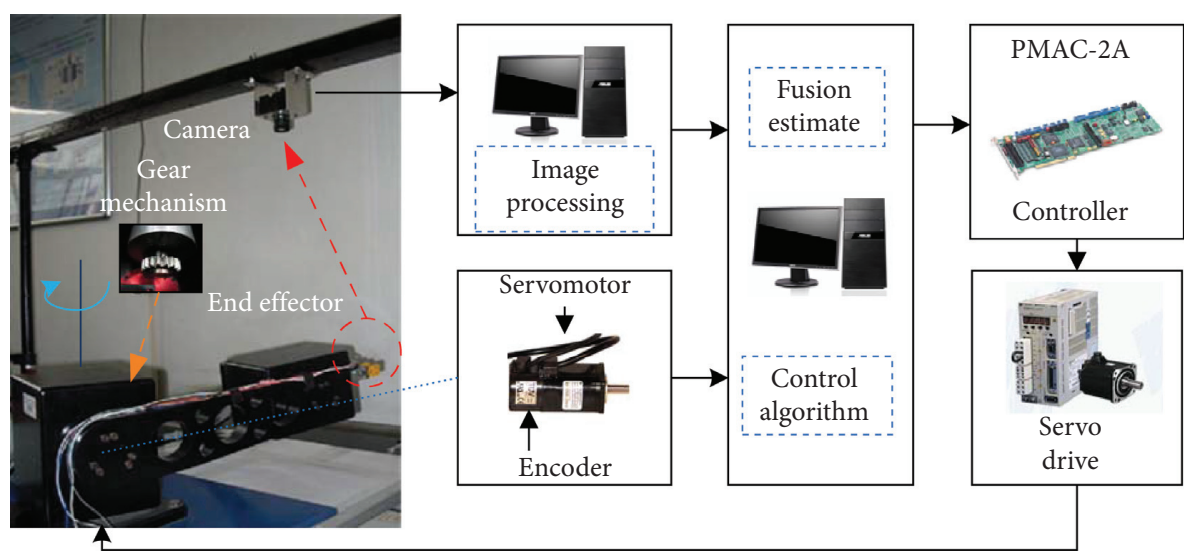

Figure 8: The experiment setup.

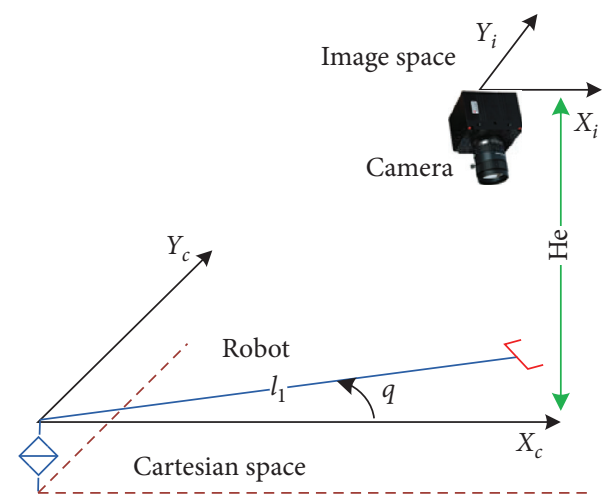

Figure 9: The coordinate relation of Cartesian space and image space.

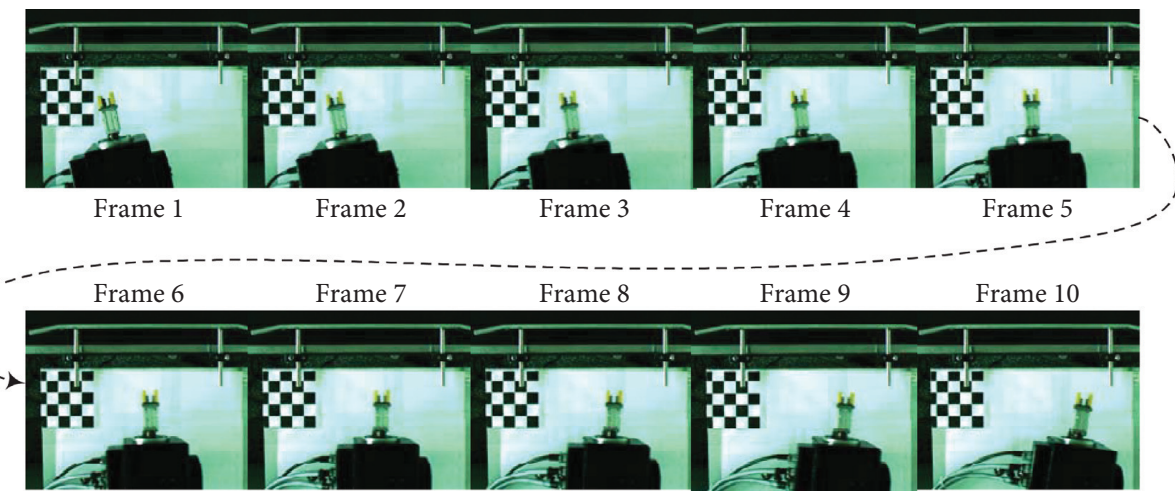

Figure 10: The image sequences of 10 frames.

is $r(k)=\left[x_{c}(k), y_{c}(k)\right]$. By using the pinhole camera model, we calculate the mapping from the joint space to image space $I_{k}(\cdot)$ in equation (14) as follows:

$$
\frac{f}{H_{c}-f}\left[\begin{array}{l}
x_{m}(k) \\
y_{m}(k)
\end{array}\right]=\left[\begin{array}{l}
x_{c}(k) \\
y_{c}(k)
\end{array}\right]=\left[\begin{array}{l}
l_{1} \cos q(k) \\
l_{2} \sin q(k)
\end{array}\right],
$$

where $H_{c}$ is the perpendicular distance between the camera and the robot motion plane and $f$ denotes the focal length of the camera. In the experiment, camera parameters are $f=0.035 \mathrm{~m}, 640 \times 512$ pixels, and $H_{c}=0.8 \mathrm{~m}$. According to the mapping $I_{k}(\cdot)$, the joint position can be obtained by calculating the inverse circular trigonometric functions $\arccos \left[x_{m}(k) f /\left(l_{1}\left(H_{c}-f\right)\right)\right]$ or $\arcsin \left[y_{m}(k) f /\left(l_{1}\left(H_{c}-\right.\right.\right.$ $f))]$. The delayed vision measurements $\left[x_{m}(k), y_{m}(k)\right]$ are converted to joint measurements $\arccos \left[x_{m}(k) f /\left(l_{1}\left(H_{c}-\right.\right.\right.$ $f)$ )] which are shown in Figure 12, where the encoder measurements are also plotted. The position estimation of 


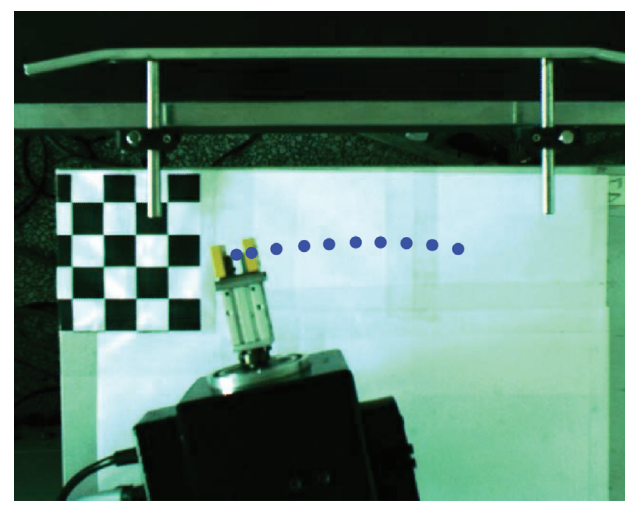

- Position measurements in image space

FIgURE 11: The trajectories of end effector in image space.

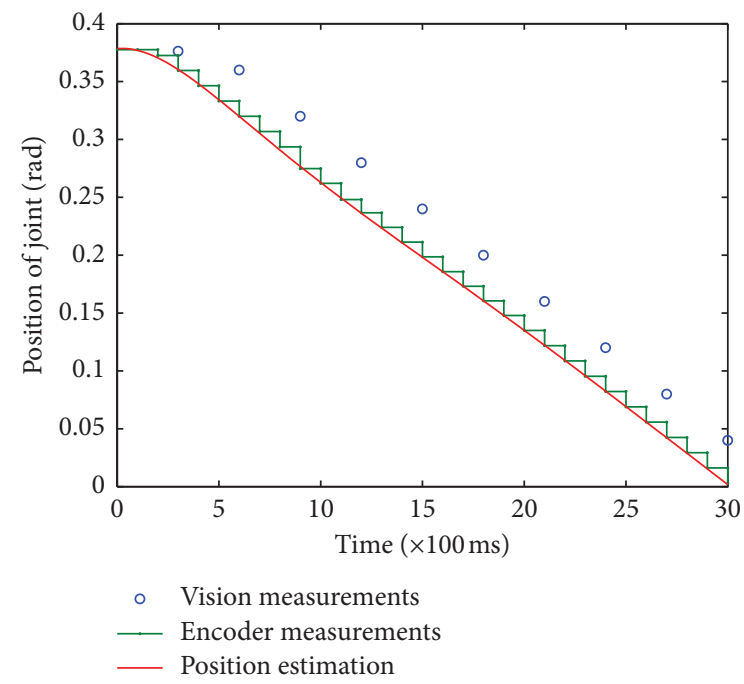

FIgURe 12: The encoder and visual measurements.

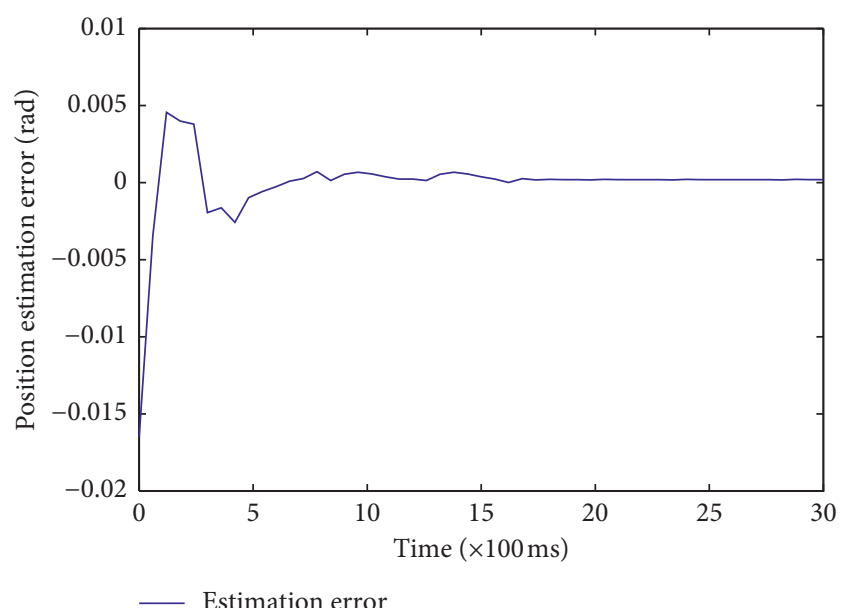

Figure 13: The position estimation error of the joint.

the joint is calculated by using the proposed fusion estimate method shown as red solid line in Figure 12. To show the
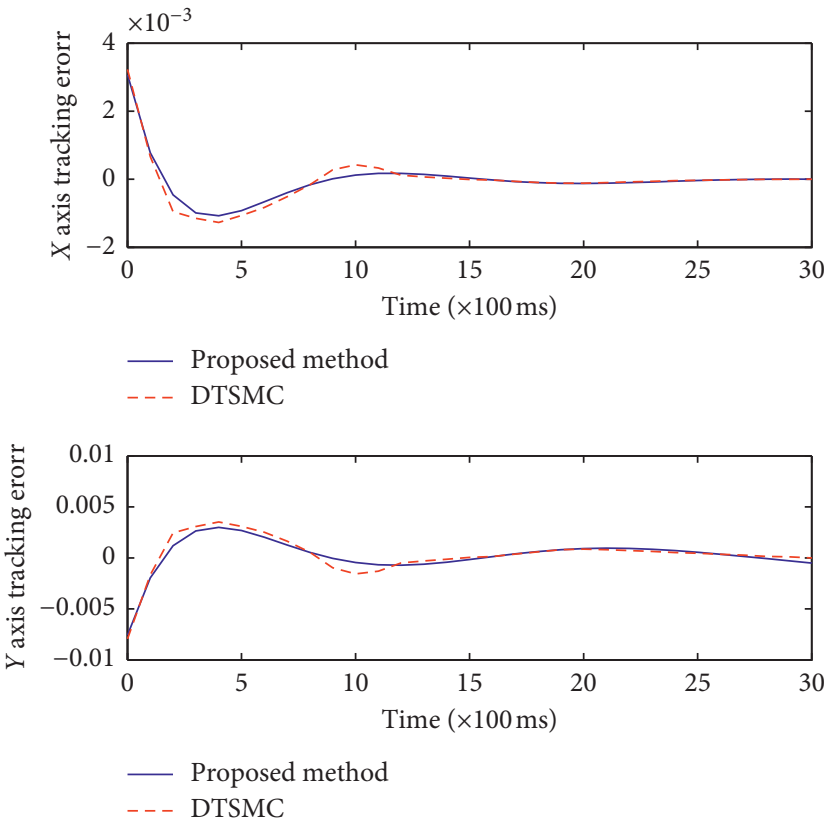

Figure 14: Comparative position tracking errors of the end effector.

performance detail of the fusion estimate method, Figure 13 shows the error of joint position estimation.

To validate the performance of the fusion estimate-based DTSMC, comparative experiments are implemented in this paper. The proposed controller and a DTSMC without estimator are employed in this test. Parameters are selected as $T=0.01 \mathrm{~s}, h=3, \lambda=2, \Lambda_{t}=4$ and $\varepsilon=20, \theta(k)=0.05$; $p=3, q=5, \xi_{\mathbb{M}}=0.2$ and $\xi_{\mathbb{F}}=2$. According to the comparative position tracking performances given in Figure 14, it is obvious that the proposed controller provides a superior behavior.

\section{Conclusion}

A novel RLFJ manipulator tracking controller, AMSFEDTSMC, is proposed in this paper based on DTSMC coupled with asynchronous multirate sensor fusion. States of the manipulator are estimated by EKF-based sensor fusion algorithm which combines asynchronous multirate measurements from visual and non-vision-based sensors. Compared with the non-vision measurements, visual measurements are considered as periodic out-of-sequence measurements, which are used to re-estimate the state. With the state estimation, DTSMC is designed by using a novel sliding surface that includes tracking error and estimation error. By using the Sarpturk inequalities, boundedness of the controlled variables is proved. The effectiveness of the proposed approach is shown in simulation and experimental studies.

\section{Data Availability}

No data were used to support this study. 


\section{Disclosure}

An earlier version of multirate sensor fusion-based DTSMC has been presented in the 10th International Conference on Control and Automation (ICCA) [12]; however, a totally new controller is designed by using a new sliding surface including both tracking error and estimation error in this paper, and the effectiveness of the proposed approach is proved both in the simulation and experimental studies.

\section{Conflicts of Interest}

The authors declare that they have no conflicts of interest.

\section{References}

[1] S. Nicosia and P. Tomei, "A tracking controller for flexible joint robots using only link position feedback," IEEE Transactions on Automatic Control, vol. 40, no. 5, pp. 885890, 1995.

[2] S. Nicosia, P. Tomei, and A. Tornambe, "A nonlinear observer for elastic robots," IEEE Journal on Robotics and Automation, vol. 4, no. 1, pp. 45-52, 1988.

[3] W. E. Dixon, E. Zergeroglu, D. M. Dawson, and M. W. Hannan, "Global adaptive partial state feedback tracking control of rigid-link flexible-joint robots," Robotica, vol. 18, no. 3, pp. 325-336, 2000.

[4] F. Abdollahi, H. A. Talebi, and R. V. Patel, "A stable neural network-based observer with application to flexible-joint manipulators," IEEE Transactions on Neural Networks, vol. 17, no. 1, pp. 118-129, 2006.

[5] J. Na, B. Jing, Y. Huang, G. Gao, and C. Zhang, "Unknown system dynamics estimator for motion control of nonlinear robotic systems," IEEE Transactions on Industrial Electronics, vol. 67, no. 5, pp. 3850-3859, 2020.

[6] S. Jean, M. Tomizuka, and T. Katou, "Kinematic kalman filter (KKF) for robot end-effector sensing," Journal of Dynamic Systems, Measurement, and Control, vol. 131, no. 2, pp. 1-8, 2009.

[7] C. A. Lightcap and S. A. Banks, "An extended kalman filter for real-time estimation and control of a rigid-link flexible-joint manipulator," IEEE Transactions on Control Systems Technology, vol. 18, no. 1, pp. 91-103, 2010.

[8] J. G. Garcła, A. Robertsson, J. G. Ortega, and R. Johansson, "Sensor fusion for compliant robot motion control," IEEE Transactions on Robotics, vol. 24, no. 2, pp. 430-441, 2008.

[9] R. Jassemi-Zargani and D. Necsulescu, "Extended kalman filter-based sensor fusion for operational space control of a robot arm," IEEE Transactions on Instrumentation and Measurement, vol. 51, no. 6, pp. 1279-1282, 2002.

[10] S. Wang, L. Tao, Q. Chen, J. Na, and X. Ren, "USDE-based sliding mode control for servo mechanisms with unknown system dynamics," IEEE/ASME Transactions on Mechatronics, vol. 25, no. 2, pp. 1056-1066, 2020.

[11] K. Jezernik, B. Curk, and J. Harnik, "Observer-based sliding mode control of a robotic manipulator," Robotica, vol. 12, no. 5, pp. 443-448, 1994.

[12] G. Xue, X. Ren, K. Xing, and Q. Chen, "Discrete-time sliding mode control coupled with asynchronous sensor fusion for rigid-link flexible-joint manipulators," in Proceedings of the 2013 10th IEEE International Conference on Control and Automation (ICCA), pp. 238-243, Hangzhou, China, June 2013.
[13] Y. Gao, S. S. Majidabad, and H. T. Shandiz, "Discrete-time based sliding-mode control of robot manipulators," International Journal of Intelligent Computing and Cybernetics, vol. 5, no. 3, pp. 340-358, 2012.

[14] M. L. Corradini, V. Fossi, A. Giantomassi, G. Ippoliti, S. Orlando, and G. Orlandob, "Discrete time sliding mode control of robotic manipulators: development and experimental validation," Control Engineering Practice, vol. 20, no. 8, pp. 816-822, 2012.

[15] L. Wang, T. Chai, and L. Zhai, "Neural-network-based terminal sliding-mode control of robotic manipulators including actuator dynamics," IEEE Transactions on Industrial Electronics, vol. 56, no. 9, pp. 3296-3304, 2009.

[16] Y. Feng, X. Yu, and Z. Man, "Non-singular terminal sliding mode control of rigid manipulators," Automatica, vol. 38, no. 12 , pp. 2159-2167, 2002.

[17] M. L. Corradini and G. Orlando, "Linear unstable plants with saturating actuators: robust stabilization by a time varying sliding surface," Automatica, vol. 43, no. 1, pp. 88-94, 2007.

[18] Y. Bar-Shalom, H. Huimin Chen, and M. Mallick, "One-step solution for the multistep out-of-sequence-measurement problem in tracking," IEEE Transactions on Aerospace and Electronic Systems, vol. 40, no. 1, pp. 27-37, 2004.

[19] K. Reif, F. Sonnemann, and R. Unbehauen, "An EKF-based nonlinear observer with a prescribed degree of stability," Automatica, vol. 34, no. 9, pp. 1119-1123, 1998.

[20] W. Gao, Y. Wang, and A. Homaifa, "Discrete-time variable structure control systems," IEEE Transactions on Industrial Electronics, vol. 42, no. 2, pp. 117-122, 1995.

[21] S. Sarpturk, S. Y. Istefanopulos, Y. Istefanopulos, and O. Kaynak, "On the stability of discrete-time sliding mode control systems," IEEE Transactions on Automatic Control, vol. 32, no. 10, pp. 930-932, 1987. 